\title{
Pattern classification of endocervical adenocarcinoma: reproducibility and review of criteria
}

Joanne KL Rutgers ${ }^{1}$, Andres A Roma ${ }^{2}$, Kay J Park ${ }^{3}$, Richard J Zaino ${ }^{4}$, Abbey Johnson ${ }^{5}$, Isabel Alvarado ${ }^{6}$, Dean Daya ${ }^{7}$, Golnar Rasty ${ }^{8}$, Teri A Longacre ${ }^{9}$, Brigitte M Ronnett ${ }^{10}$ and Elvio G Silva ${ }^{11}$

${ }^{1}$ Department of Pathology, Cedars-Sinai Medical Center, Los Angeles, CA, USA; ${ }^{2}$ Department of Pathology, Cleveland Clinic, Cleveland, OH, USA; ${ }^{3}$ Department of Pathology, Memorial Sloan Kettering Cancer Center, New York, NY, USA; ${ }^{4}$ Department of Pathology, Pennsylvania State University, State College, PA, USA; ${ }^{5}$ Department of Pathology, Corpath, Columbus, OH, USA; ${ }^{6}$ Hospital de Oncología Centro Médico Nacional Siglo XXI, Ciudad de México, D.F., Mexico; ${ }^{7}$ Department of Pathology, Juravinski Hospital, Hamilton, ONT, Canada; ${ }^{8}$ Department of Pathology, Toronto East General Hospital, Toronto, ONT, Canada; ${ }^{9}$ Department of Pathology, Stanford University School of Medicine, Stanford, CA, USA; ${ }^{10}$ Department of Pathology, Johns Hopkins University School of Medicine, Baltimore, MD, USA and ${ }^{11}$ Department of Pathology, MD Anderson Center, Houston, Texas, and Cedars-Sinai Medical Center, Los Angeles, CA, USA

Previously, our international team proposed a three-tiered pattern classification (Pattern Classification) system for endocervical adenocarcinoma of the usual type that correlates with nodal disease and recurrence. Pattern Classification-A tumors have well-demarcated glands lacking destructive stromal invasion or lymphovascular invasion, Pattern Classification-B tumors show localized, limited destructive invasion arising from A-type glands, and Pattern Classification-C tumors have diffuse destructive stromal invasion, significant (filling a $4 \times$ field) confluence, or solid architecture. Twenty-four cases of Pattern Classification-A, 22 Pattern Classification-B, and 38 Pattern Classification-C from the tumor set used in the original description were chosen using the reference diagnosis originally established. One H\&E slide per case was reviewed by seven gynecologic pathologists, four from the original study. Kappa statistics were prepared, and cases with discrepancies reviewed. We found a majority agreement with reference diagnosis in $81 \%$ of cases, with complete or near-complete (six of seven) agreement in $\mathbf{5 0} \%$. Overall concordance was $\mathbf{7 4 \%}$. Overall kappa (agreement among pathologists) was 0.488 (moderate agreement). Pattern Classification-B has lowest kappa, and agreement was not improved by combining $B+C$. Six of seven reviewers had substantial agreement by weighted kappas $(>0.6)$, with one reviewer accounting for the majority of cases under or overcalled by two tiers. Confluence filling a $4 \times$ field, labyrinthine glands, or solid architecture accounted for undercalling other reference diagnosis-C cases. Missing a few individually infiltrative cells was the most common cause of undercalling reference diagnosis-B. Small foci of inflamed, loose or desmoplastic stroma lacking infiltrative tumor cells in reference diagnosis-A appeared to account for those cases up-graded to Pattern Classification-B. In summary, an overall concordance of $74 \%$ indicates that the criteria can be reproducibly applied by gynecologic pathologists. Further refinement of criteria should allow use of this powerful classification system to delineate which cervical adenocarcinomas can be safely treated conservatively. Modern Pathology (2016) 29, 1083-1094; doi:10.1038/modpathol.2016.94; published online 3 June 2016

Endocervical adenocarcinoma accounts for $15-20 \%$ of cervical carcinoma, is increasing in incidence, and often occurs in young women. ${ }^{1}$ Recently, our

Correspondence: Dr JKL Rutgers. MD, Department of Pathology, Cedars-Sinai Medical Center, 8700 Beverly Blvd, Los Angeles, CA 90048, USA.

E-mail: joanne.rutgers@cshs.org

Received 11 February 2016; revised 7 April 2016; accepted 14 April 2016; published online 3 June 2016 international collaborative team proposed a novel histopathologic pattern classification system for endocervical adenocarcinoma that strongly correlates with the risk of nodal metastases and recurrence. ${ }^{2,3}$ Pattern Classification classifies endocervical adenocarcinoma into one of three patterns based on the degree of destructive stromal invasion and lymphovascular invasion, regardless of the depth of invasion. Pattern Classification-A tumors have a 
non-destructive pattern of stromal invasion without lymphovascular invasion. Pattern Classification-B have early, small foci of destructive stromal invasion typically arising from Pattern Classification-A type glands, and may show lymphovascular invasion. Pattern Classification-C tumors have diffuse destructive invasion, confluent patterns of invasion filling a $4 \times$ field, or high architectural grade (solid growth), and frequent lymphovascular invasion. In the two sets of patients studied to date including over 400 patients ${ }^{3,4}$ Pattern Classification-A has not shown nodal metastases or recurrences, Pattern Classification-B has $<5 \%$ risk of nodal metastases or recurrences, with those cases with positive nodes only found in tumors with lymphovascular invasion, and Pattern Classification-C captures those endocervical adenocarcinoma with a high rate, approximately $25 \%$, of nodal metastases and recurrences. Pattern Classification-A and -B have only been identified in FIGO stage I tumors, with an approximately equal distribution between FIGO stages IA and IB. In contrast, $15 \%$ of Pattern Classification-C tumors were stage II or higher. These studies suggest that Pattern Classification-A endocervical adenocarcinoma can be safely treated by excision with negative margins without nodal sampling, and likely do not need adjuvant therapy following surgery, Pattern Classification-C may need aggressive therapy, and treatment of Pattern Classification-B may be individualized by the presence or absence of lymphovascular invasion.

FIGO staging, the presence or absence of lymphovascular invasion, tumor size $>2 \mathrm{~cm}$, and proportion of cervical wall thickness involved by tumor currently guide treatment of endocervical adenocarcinoma. ${ }^{5}$ FIGO staging applies to both squamous cell carcinoma and endocervical adenocarcinoma of the cervix. When surgical therapy is undertaken, the National
Comprehensive Cancer Network treatment guidelines mandate radical surgery with pelvic nodal dissection for stage IA1 with lymphovascular invasion and any tumor stage IA2 or higher, albeit with consideration of fertility sparing surgery. ${ }^{6}$ The staging is a hybrid of clinical examination and pathologic examination of the tumor, with clinically visible tumors staged IB, and those tumors only identified by microscopic examination are staged ${ }^{7}$ according to depth of invasion and horizontal extent of tumor. Thus, it is essential to accurately measure depth of invasion, which is intended to measure the depth of invasion from the basement membrane of the epithelium from which the invasion arose. It is often much easier for the pathologist to measure depth of invasion for squamous cell carcinoma; for instance if a small tongue of invasive disease arises from an endocervical gland involved by squamous carcinoma in situ (highgrade squamous intraepithelial lesion, HSIL) only that group of cells will be measured, even when there is extensive HSIL. In contrast, determining which gland from which a small endocervical adenocarcinoma arose can be difficult, and for endocervical adenocarcinoma many suggest measuring depth of invasion from the basement membrane of the surface epithelium, ${ }^{8,9}$ a method that renders depth of invasion equivalent to tumor thickness. This practice may upstage endocervical adenocarcinoma when the invasive component is admixed with endocervical adenocarcinoma in situ (adenocarcinoma in situ).

Studies suggest that the Pattern Classification better identifies those tumors with a low to no risk of nodal metastases and recurrences than FIGO substaging. ${ }^{2-4}$ Application of Pattern Classification to clinical practice mandates that pathologists can use it reproducibly. Moderate agreement (mean pairwise kappa value of 0.51) was achieved in a prior study of 49 endocervical adenocarcinoma. ${ }^{4}$

Table 1 Histologic criteria of the three Pattern Classifications

\begin{tabular}{|c|c|}
\hline Pattern classification & Criteria \\
\hline \multirow[t]{7}{*}{ Pattern Classification-A } & Well-demarcated glands with rounded contours, frequently forming groups \\
\hline & No destructive stromal invasion \\
\hline & No single cells or cell detachment \\
\hline & No lymphovascular invasion \\
\hline & Complex intraglandular growth acceptable, that is, cribriform, papillae \\
\hline & Lack of solid growth, that is, architecturally well-moderately differentiated \\
\hline & Depth of the tumor or relationship to large cervical vessels not relevant \\
\hline \multirow[t]{5}{*}{ Pattern Classification-B } & Localized (limited, early) destructive stromal invasion arising from pattern A (well-demarcated) glands \\
\hline & $\begin{array}{l}\text { Individual or small groups of tumor cells, separated from the rounded gland, often in a focally } \\
\text { desmoplastic or inflamed stroma }\end{array}$ \\
\hline & Foci may be single, multiple, or linear at base of tumor \\
\hline & Lymphovascular invasion \pm \\
\hline & Lack of solid growth, that is, architecturally well-moderately differentiated \\
\hline \multirow[t]{4}{*}{ Pattern Classification-C } & $\begin{array}{l}\text { Diffuse destructive stromal invasion, characterized by diffusely infiltrative glands, with associated } \\
\text { extensive desmoplastic response }\end{array}$ \\
\hline & Confluent growth filling a $4 \times$ field ( $5 \mathrm{~mm}$ ): glands, papillae (stroma only within papillae), or mucin lakes \\
\hline & Glands often angulated or with a canalicular pattern, with interspersed open glands \\
\hline & Solid, poorly differentiated component (architecturally high grade); nuclear grade is disregarded \\
\hline
\end{tabular}


The goal of this study is to further test the reproducibility in a larger study using a subset of the original cases, ${ }^{2,3}$ and examine those cases with variability to clarify and illustrate the Pattern Classification criteria.

\section{Materials and methods}

Eighty-four cases were chosen from the original group of 352 to include 24 cases of Pattern Classification-A, 22 Pattern Classification-B, and 38 Pattern Classification-C. Cases were chosen to achieve an approximate equal distribution of the three pattern classifications as in the original study, to include cases so that each criteria will need to be used, and to choose cases with the highest quality and representative single $\mathrm{H} \& \mathrm{E}$ slide. The reference diagnosis was established by the original studies that included follow-up information, with arbitration of difficult cases by the first and senior authors, in the absence of a 'gold standard'. One H\&E slide per case was distributed to seven gynecologic pathologists, four from the original group. There was a time delay of more than a year between the original review and this study, in an attempt to obviate bias among the original group. The reviewers were provided with reproductions of our second paper, including a table listing criteria (Table 1), clues to distinguish Pattern Classification-A from Pattern Classification-C (Table 2), ${ }^{3}$ and additional hints for distinguishing

Table 2 Features to distinguish Pattern Classification A from Pattern Classification C

\begin{tabular}{lll}
\hline & Pattern $A$ & Pattern $C$ \\
\hline Diffuse desmoplasia & No & Yes \\
Gland contours & Round & Angulated \\
Interspersed open glands $^{\mathrm{a}}$ & No & Yes \\
Cluster or groups of glands $_{\text {Canalicular pattern }}^{\mathrm{b}}$ & Yes & No \\
& No & Yes \\
\hline
\end{tabular}

${ }^{\mathrm{a}}$ Open glands (incomplete glandular structures) describe glands with a discontinuous contour showing a break opening to the stroma, often associated with loosened stroma and/or inflammatory cells. ${ }^{\mathrm{b}}$ Canalicular pattern means labyrinthine, interconnected glands.
Pattern Classification-A from Pattern Classification-B (Figure 1).

Statistical analysis included a seven rater (overall) kappa, simple and weighted kappas and Spearman correlation coefficient for each reviewer, and Pattern Classification-A vs Pattern Classification-B+C kappa statistics, performed by a biostatistician using SAS version 9.2 (SAS Institute, Cary, NC, USA). Kappa of 0-0.2 is slight, 0.2-0.4 fair, 0.4-0.6 moderate, 0.6-0.8 substantial, and $0.8-1$ almost perfect agreement. ${ }^{10}$ The proportions of diagnoses overinterpreted or underinterpreted, by one or two steps, relative to the reference diagnosis were determined. ${ }^{11}$

After results and comments from the reviewers were collated, problematic cases, including those with majority disagreement with the reference diagnosis, those in which there were choices from all three categories, or those with a discrepancy of two tiers were re-reviewed by the first and senior authors.

\section{Results}

The majority of reviewers agreed with the reference diagnosis in $68(81 \%)$ cases, with complete or nearcomplete (6/7) agreement in 50\%. Examples of cases with excellent concordance are illustrated in Figure 2.

Correlation statistics calculated for Pattern Classification-A vs Pattern Classification-B vs Pattern Classification-C are provided for each of the reviewers; $\mathrm{P} 1$ to $\mathrm{P} 4$ were pathologists from the original group (Table 3). The simple kappa (nonordinal test) showed substantial agreement by three reviewers, and moderate by four. Weighted kappa showed substantial agreement by six reviewers $(86 \%)$ and moderate agreement by one reviewer. Spearman's correlation coefficient ranged from 0.581 to 0.853 . The overall kappa of 0.49 represents moderate agreement. Overall kappa for Pattern Classification-A was 0.54, for Pattern ClassificationB 0.32, and for Pattern Classification-C 0.59, indicating the greatest reproducibility for Pattern Classification-C. Agreement is more than can be expected from chance $(P<0.0001)$ for each kappa. Combining Pattern Classification- $\mathrm{B}+\mathrm{C}$ achieves the

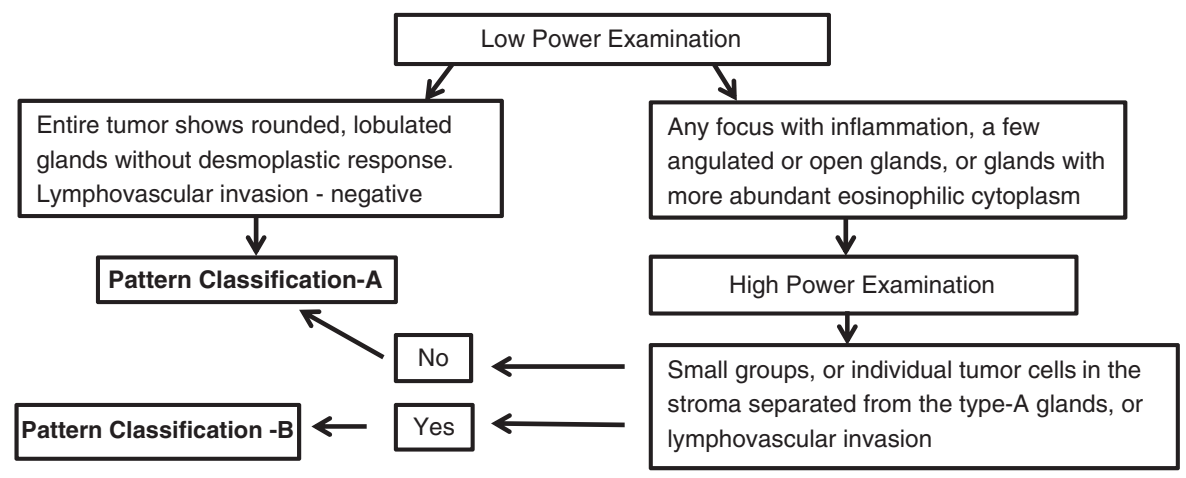

Figure 1 Flow sheet to distinguish Pattern Classification-A from Pattern Classification-B. 

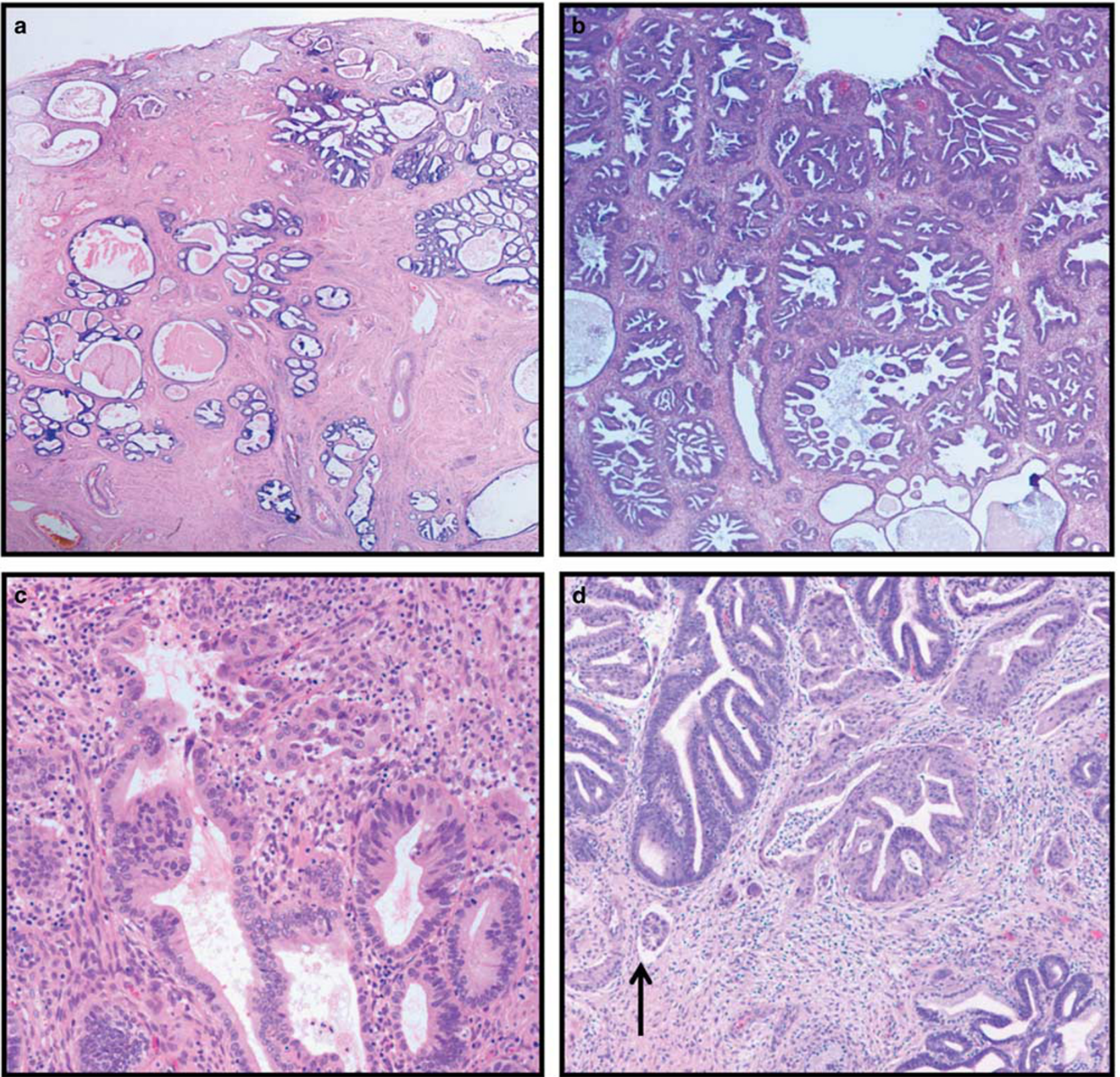

Figure 2 Cases with excellent reproducibility. (a) Low power of reference diagnosis-A correctly diagnosed by all seven reviewers. This tumor has deep invasion lacking a stromal response, with lobulated groups of rounded glands, some adjacent to thick-walled vessels (original magnification $\times 2$ ). (b) Another reference diagnosis-A correctly diagnosed by all reviewers. There is intraglandular complexity but the shape of each gland in relation to the stroma is rounded. There is no stromal response (original magnification $\times 4$ ). (c) This reference diagnosis-B tumor was correctly diagnosed by five of seven reviewers. There are small buds of infiltrating tumor cells, with slightly more abundant, eosinophilic cytoplasm arising from type A glands. The focus of destructive invasion also shows an inflammatory infiltrate (original magnification $\times 20$ ). (d) This reference diagnosis-B tumor was correctly diagnosed by six of seven reviewers. There are a variety of patterns, with adenocarcinoma in situ lower right, type A glands upper left, type B glands on the right, and lymphovascular invasion (arrow) (original magnification $\times 10$ ). (e) Many Pattern Classification-C tumors can be easily recognized immediately by both diffuse destructive invasion with extensive desmoplasia and confluence; this tumor was diagnosed as Pattern Classification-C by all seven reviewers (original magnification $\times 4$ ). (f) Pattern Classification-C tumors may be smaller than a $4 \times$ field, but show an extensive desmoplastic response filling all of the stroma between the glands; this case was called Pattern Classification-C by six of seven reviewers (original magnification $\times 4$ ).

same kappa, 0.54 as for Pattern ClassificationA alone.

The distribution of scoring opportunities (Table 4) shows agreement with the reference diagnosis in 129 of $168(77 \%)$ of reference diagnosis-A, 104 of 154 (68\%) of reference diagnosis-B, and 205 of 266 (77\%) of reference diagnosis-C, for an overall concordance of $74 \%$. The six scoring opportunities of reference diagnosis-A called Pattern Classification-C were six cases all from one reviewer and represent $3 \%$ of scoring opportunities. The 16 scoring opportunities of reference diagnosis-C under-called by two steps 

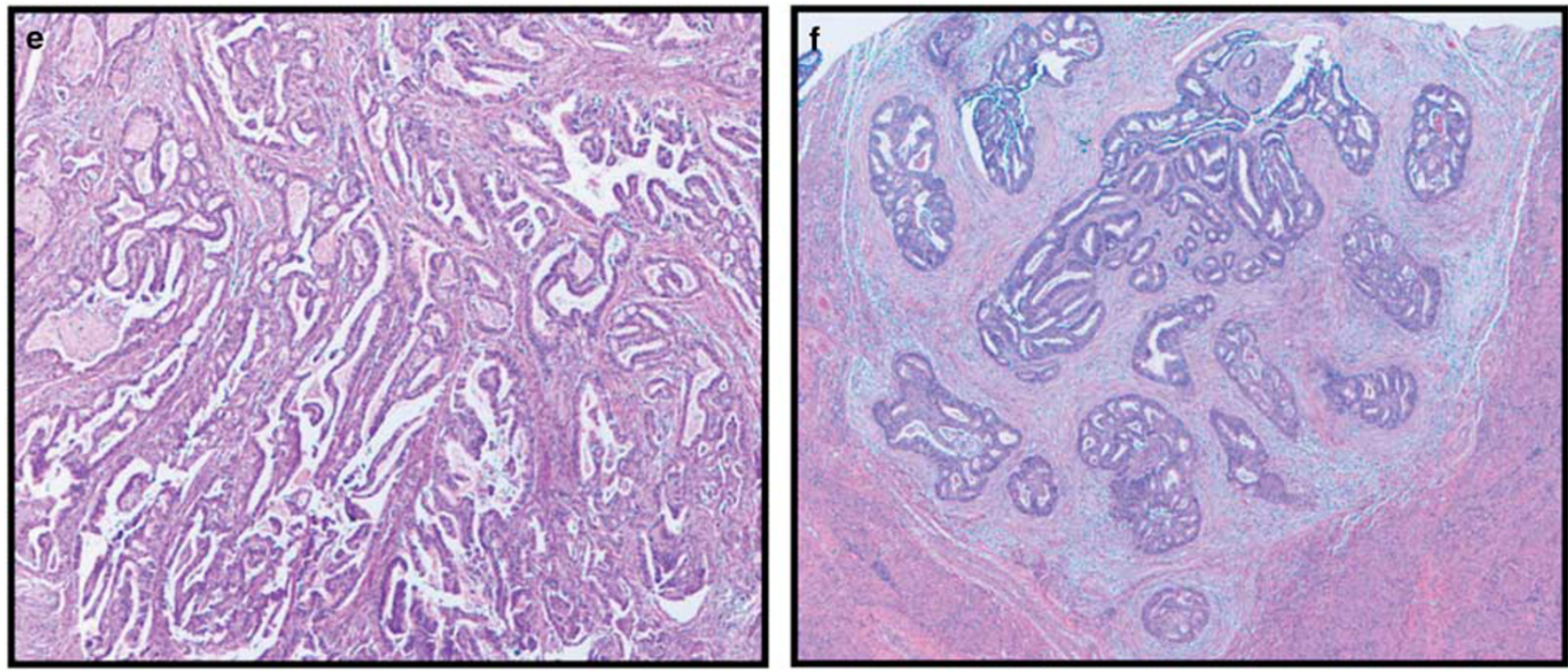

Figure 2 (Continued).

Table 3 Correlation statistics

\begin{tabular}{lccc}
\hline Pathologist no. & $\begin{array}{c}\text { Simple } \\
\text { kappa }\end{array}$ & $\begin{array}{c}\text { Weighted } \\
\text { kappa }\end{array}$ & $\begin{array}{c}\text { Spearman } \\
\text { correlation }\end{array}$ \\
\hline 1 & 0.710 & 0.781 & 0.853 \\
2 & 0.681 & 0.737 & 0.808 \\
3 & 0.549 & 0.522 & 0.581 \\
4 & 0.529 & 0.602 & 0.717 \\
5 & 0.704 & 0.779 & 0.867 \\
6 & 0.595 & 0.640 & 0.700 \\
7 & 0.535 & 0.635 & 0.719 \\
\hline
\end{tabular}

were six cases under-called by various reviewers, representing $6 \%$ of reference diagnosis-C scoring opportunities. Thus, the scoring opportunity data indicate a $9 \%$ significant $(2=$ tier $)$ under- or overinterpretation rate.

There were 16 cases in which the majority of pathologists did not agree with the reference diagnosis, including four reference diagnosis-A, four reference diagnosis- $B$, and eight reference diagnosis-C. These cases and those cases misclassified by two steps underwent intensive review of the glass slides to determine potential reasons for discrepancy. The most common reason identified for undercalling reference diagnosis- $\mathrm{C}$ was to not apply the criterion of confluent tumor filling a $4 \times$ field (Figure 3a). The term confluent has been used for different patterns over time, but in this context indicates a lack of stroma separating the malignant glands, or confluence of tumor papillae, with stroma only within the papillary cores. These confluence criteria are not intended to apply to the exophytic papillary component, ${ }^{3}$ but this distinction proved difficult in several cases (Figures $3 \mathrm{~b}$ and $\mathrm{c}$ ). A canalicular, or labyrinthine growth pattern, is a criterion for Pattern Classification-C even if the tumor is smaller than a $4 \times$ field (Figure $3 \mathrm{~d}$ ).
Non-recurrent issues were also identified. One reference diagnosis-C case with subtle diffuse infiltration but without a significant desmoplastic response nevertheless had extensive lymphovascular invasion, lack of clustering of glands, and focal angulated glands (Figures $4 \mathrm{a}$ and b). The one reference diagnosis- $C$ case with a solid architecture included in this study proved controversial, with only one reviewer classifying as Pattern Classification-C and 6 as Pattern Classification-A (Figure 4c). When a linear area of destructive invasion exceeds a $4 \times$ field, it is classified as Pattern Classification-C; not applying these criteria resulted in undercalling a few reference diagnosis-C cases (Figure 4d). A final factor identified was difficulty in interpretation due to a partially obscuring inflammatory infiltrate.

Early destructive invasion in a Pattern Classification-B can be focal, multifocal, or a linear area at the advancing front of the tumor measuring less than a $4 \times$ field, that is $<5 \mathrm{~mm}$. Missing a small focus of individual cells or small detached fragments of adenocarcinoma cells appeared to be the cause of the $20 \%$ of scoring opportunities in which reference diagnosis-B was classified as Pattern Classification-A.

Six reference diagnosis-A cases were called Pattern Classification-C by one reviewer; one possible reason is that the lobulation characteristic of Pattern Classification-A may be subtle (Figure 5a). Another reference diagnosis-A tumor with branched, irregularly sized glands, but overall rounded shape also proved difficult (Figure 5b). Reference diagnosis-A cases with minor foci of inflamed, loose, or desmoplastic stroma without infiltrative tumor cells accounted for up-grading to Pattern Classification-B in a few cases (Figure 5c). The intended meaning of the criterion of a desmoplastic response is spindled, loose stroma that contains individual or small detached groups of adenocarcinoma cells. Thus, Pattern Classification-A tumors may show minor, 
Table 4 Distribution of scoring opportunities by reference diagnosis

7 pathologists' diagnoses of 84 cases

\begin{tabular}{lcrr} 
& $\begin{array}{c}\text { Pattern Classification A, } \\
\text { no. (\%) }\end{array}$ & $\begin{array}{c}\text { Pattern Classification B, } \\
\text { no. (\%) }\end{array}$ & $\begin{array}{c}\text { Pattern Classification C, } \\
\text { no. (\%) }\end{array}$ \\
\hline Reference diagnosis-A (24 cases) & $129(77)$ & $33(20)$ & $6(3)$ \\
Reference diagnosis-B (22 cases) & $31(20)$ & $104(68)$ & $19(12)$ \\
Reference diagnosis-C (38 cases) & $16(6)$ & $45(17)$ & 168 \\
Total & 180 & 179 & $205(77)$ \\
2 & 589
\end{tabular}
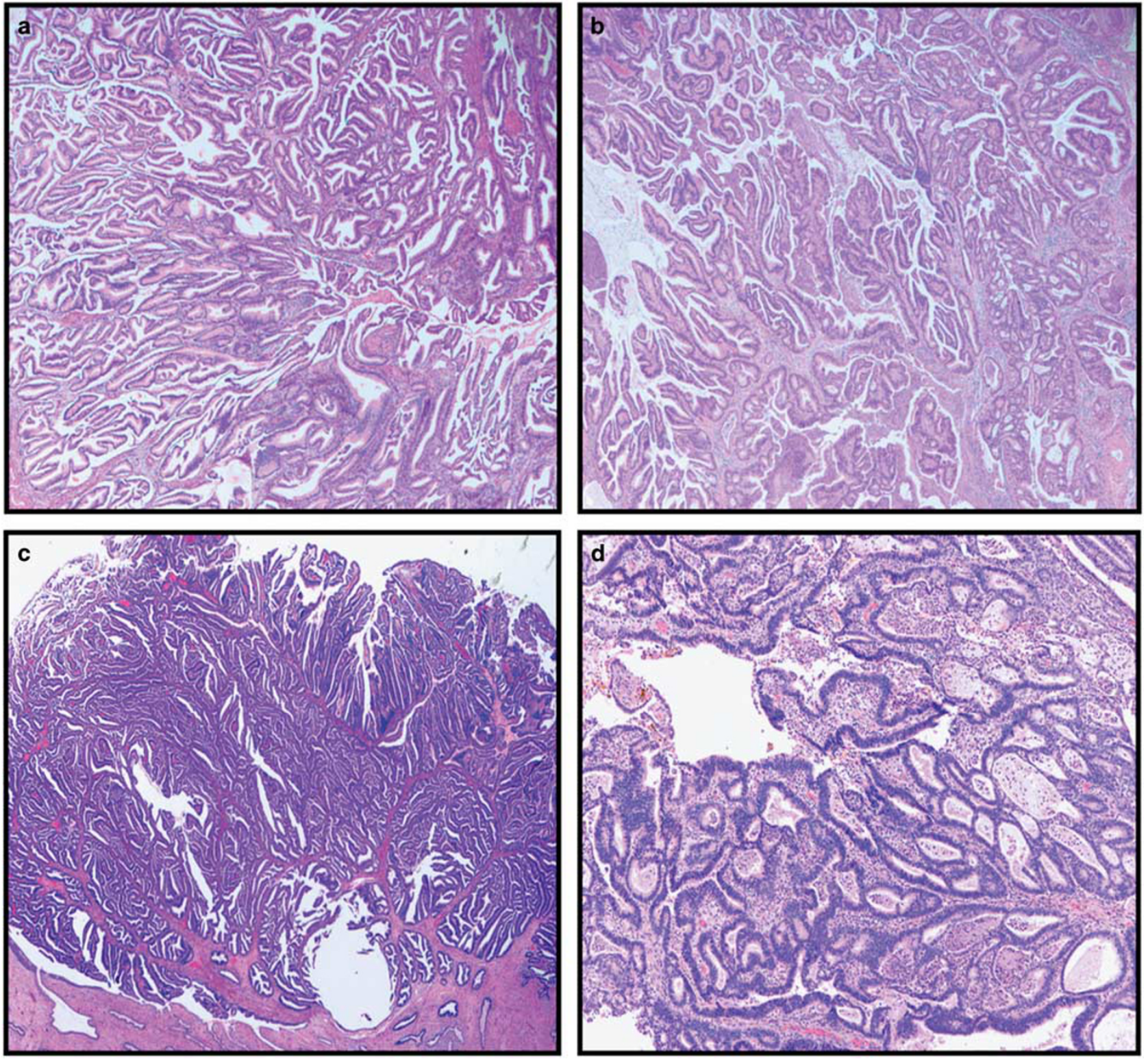

Figure 3 Cases of reference diagnosis-C without full concurrence. (a) Confluent growth filling a low power field ( $4 \times$ ) is seen in this reference diagnosis-C; this case was called Pattern Classification-B by five reviewers, and Pattern Classification-C by two (original magnification $\times 4$ ). (b) This reference diagnosis-C case was called Pattern Classification-B by four and Pattern Classification-C by three reviewers. There is both confluence of glands and papillae at the luminal aspect (left), as well as an inflamed, desmoplastic stromal response in the lower right (original magnification $\times 4$ ). (c) This polypoid tumor with confluence only towards the luminal portion of the tumor, a reference diagnosis-C due to confluence filling a $4 \times$ field was called Pattern Classification-C by only three reviewers (original magnification $\times 2$ ). Further experience with exophytic tumors is necessary. (d) This reference diagnosis-C tumor was called Pattern Classification-A by 2, Pattern Classification-B by 3, and Pattern Classification-C by 2 reviewers, respectively. This small tumor shows a labyrinthine pattern and a spindled, desmoplastic and inflamed stroma (original magnification $\times 10$ ). 

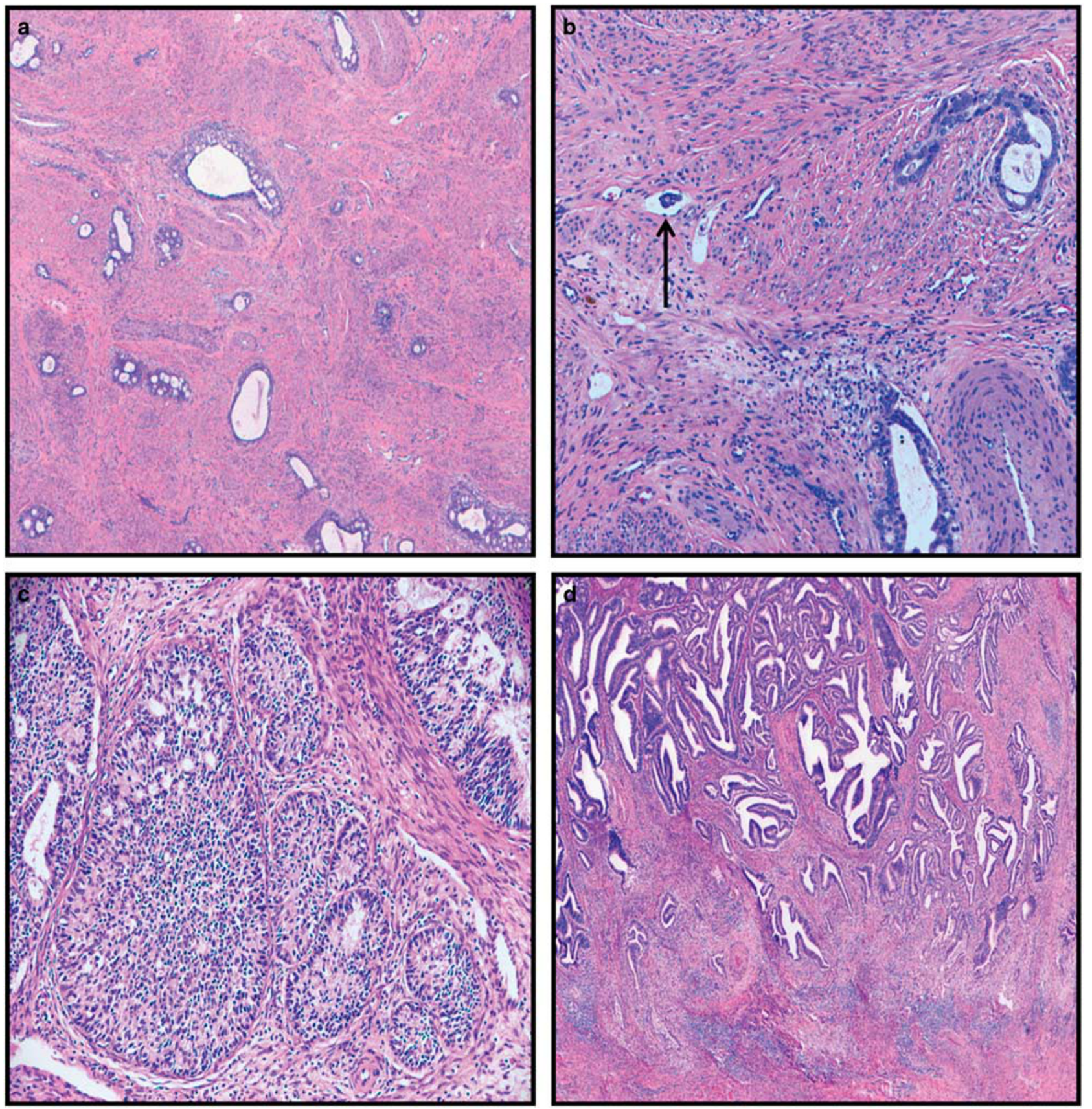

Figure 4 Additional cases of reference diagnosis-C without full concurrence. (a) Reference diagnosis-C tumor called Pattern ClassificationA by one, Pattern Classification-B by three, and Pattern Classification-C by three reviewers, respectively. The tumor demonstrates small, often angulated glands irregularly sprinkled in the stroma lacking a lobulated architecture (original magnification $\times 4$ ). (b) The same tumor as in (a) with lymphovascular invasion (arrow) and angulated gland (original magnification $\times 20$ ). (c) Reference diagnosis-C case classified as Pattern Classification-C by one and Pattern Classification-A by six reviewers. The tumor has a predominantly solid growth pattern, meeting criteria for solid growth in Pattern Classification-C, despite the rounded interface of the tumor nests with the stroma (original magnification $\times 10$ ). (d) Reference diagnosis-C due to a linear area of destructive invasion at the base of the tumor exceeding a $4 \times$ field $(>5 \mathrm{~mm}$ ). This case was called Pattern Classification-B by four and Pattern Classification-C by three reviewers, respectively (original magnification $\times 4$ ).

focal desmoplasia, loosening of the stroma, or an inflammatory response so long as these foci do not contain individual or small groups of infiltrative tumor cells; this distinction requires a high-power examination.
The comments were collated for recurrent issues. One reviewer in particular entertained adenocarcinoma in situ rather than an invasive tumor in 11 reference diagnosis-A cases, whereas the other reviewers predominantly classified these cases as 

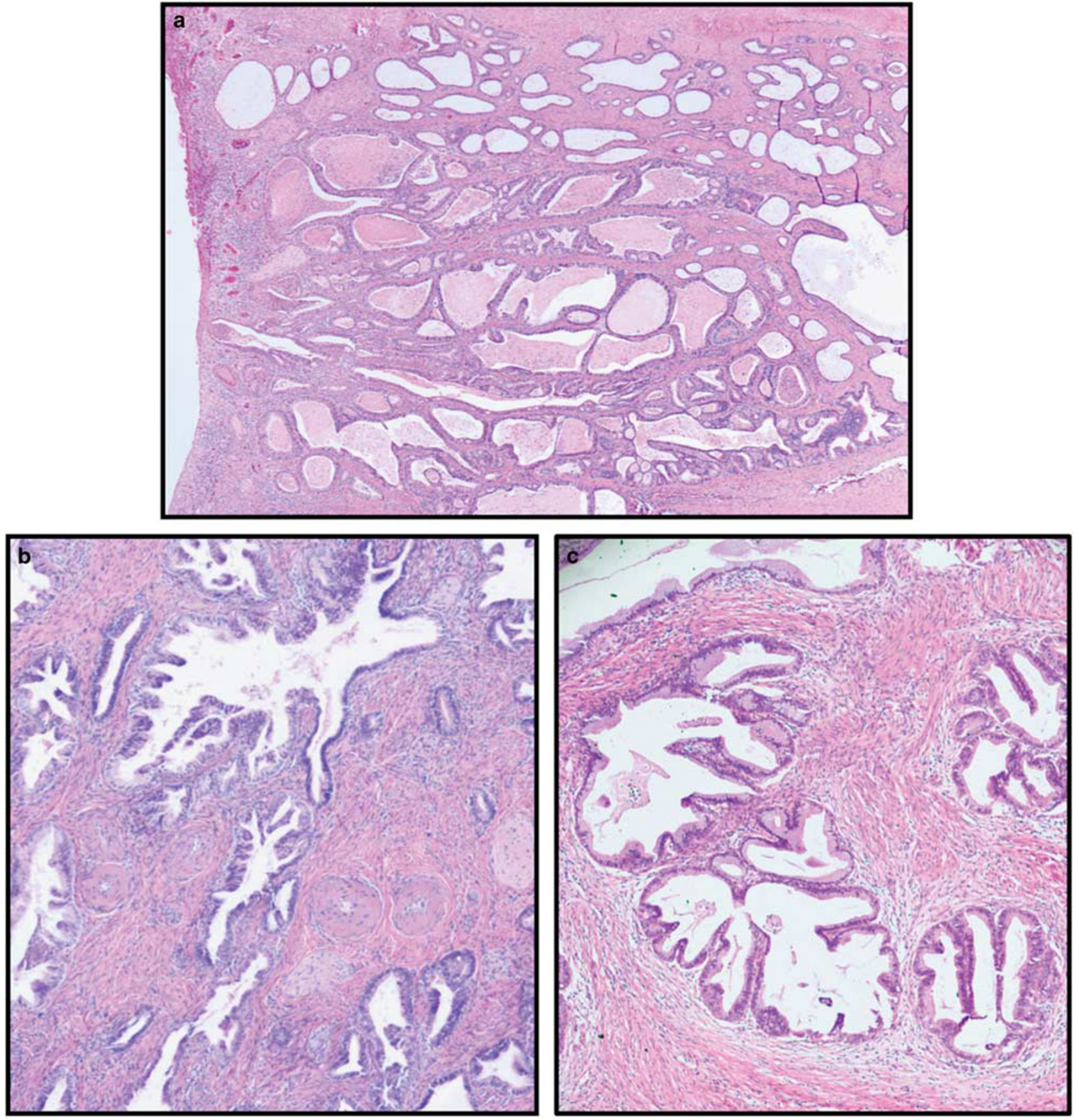

Figure 5 Reference diagnosis-A cases without full concurrence. (a) Reference diagnosis-A tumor called Pattern Classification-A by two, Pattern Classification-B by four, and Pattern Classification-C by one reviewer, respectively. At low power a lobulated architecture by rounded glands can be appreciated. There is crowding and surface inflammation but no individual infiltrative cells were present (original magnification $\times 4$ ). (b) Reference diagnosis-A tumor called Pattern Classification-A by five, Pattern Classification-B by one, and Pattern Classification-C by one reviewer, respectively. There are deeply invasive glands adjacent to thick-walled vessels and small nerve twigs. Although the glands are branched they are generally rounded (original magnification $\times 10$ ). (c) This reference diagnosis-A case was called Pattern Classification-A by three and Pattern Classification-B by four reviewers. Loosening may be present focally surrounding Pattern A type glands, but without infiltrative individual or small groups of tumor cells within the loosened stroma (original magnification $\times 10$ ).

Pattern Classification-A. Several reviewers indicated that in practice, in particular for Pattern Classification-B cases, they would have ordered additional levels. There was considerable controversy about the application of confluent growth only when located in the polypoid or luminal aspect of the tumor. A villoglandular pattern was suggested by at least one reviewer in five cases, including one with reference diagnosis of $\mathrm{A}$, one $\mathrm{B}$, and three $\mathrm{C}$. Other comments related to the precise cutoff between focal/ limited vs diffuse invasion distinguish Pattern Classification-B from Pattern Classification-C; thus 
these comments indicate issues on the application of the quantitative criteria.

\section{Discussion}

There are two competing views of endocervical adenocarcinoma, first that it has a more aggressive and unpredictable behavior than the more common squamous cell carcinoma. ${ }^{12,13}$ In addition, although the majority of endocervical adenocarcinoma are related to high-risk HPV, rare non-HPV-related subtypes including clear cell, minimal deviation, and gastric ${ }^{14-18}$ can be difficult to recognize and are aggressive. Thus, in practice there is a tendency to accept radical surgery and nodal dissection even for FIGO stage IA1 tumors lacking lymphovascular invasion. ${ }^{19}$ Alternatively, the opposing viewpoint is that endocervical adenocarcinoma do not have a worsened prognosis compared with squamous cell carcinoma, ${ }^{19}$ and that identifying reproducible criteria for which patients can be treated less aggressively is urgently needed. ${ }^{20-22}$ Studies suggest that Pattern Classification provides a safe and reproducible means to predict the risks of nodal disease and recurrence for endocervical adenocarcinoma. ${ }^{2-4}$

The gynecologic pathologists in our study were able to correctly identify $77 \%$ of reference diagnosis$\mathrm{C}$, which is in agreement with our initial experience that most Pattern Classification-C tumors can be easily recognized by the irregular infiltrative glands associated with an extensive desmoplastic response. The recognition of labyrinthine glands, solid architecture, or confluence especially in the application of one of the size-related criteria proved somewhat more difficult. The criterion of confluent growth filling a $4 \times$ field, composed of glands, papillae (stroma only within papillae), or mucin lakes containing tumor cells can be viewed as a means to detect Pattern Classification-C tumors that may lack an obvious desmoplastic response. The other quantitative criterion for Pattern Classification-C, a linear focus of destructive invasion at the base or advancing front of the tumor filling the diameter of a $4 \times$ field ( $>5 \mathrm{~mm}$ ), is intended to provide a quantitative guideline for the amount of destructive invasion to distinguish Pattern Classification-B from Pattern Classification-C, although further study of the sizerelated criteria may be necessary. Additional studies on the individual criteria for Pattern Classification-C are underway to allow refinement of the criteria.

Pattern Classification-B tumors describe those endocervical adenocarcinoma that would be Pattern Classification-A except for early, focal destructive stromal invasion that may be single, multifocal, or linear $<5 \mathrm{~mm}$ at the tumor base, and/or lymphovascular invasion. The current study found the least degree of reproducibility for reference diagnosis-B tumors. In $20 \%$ of scoring opportunities they were underclassified as Pattern Classification-A, although it should be noted that this may be due in part to the study design, in which only a single $\mathrm{H} \& \mathrm{E}$ slide was available for review. In cases lacking lymphovascular invasion, this would not be expected to affect outcome; however, lymphovascular invasion was overlooked in a few cases, making the point that careful screening for lymphovascular invasion is essential.

Pattern Classification-A shows rounded glands lined by columnar cells with pseudostratified cells and no or only focal stromal response, with no cellular budding or individual cells in the stroma. The presence of lymphovascular invasion must be searched for particularly at the edges of the tumor as lymphovascular invasion disqualifies a tumor from Pattern Classification-A. It is important to emphasize that Pattern Classification-A tumors may show deep invasion, and on a biopsy, excisional specimen, or cone biopsy, a definitive Pattern Classification-A or Pattern Classification-B cannot be assigned when the margin is positive. Additionally, tumors with a predominant Pattern Classification-A pattern but with associated desmoplasia, inflammation, localized edema, or broken glands need to be carefully examined, perhaps with multiple levels, to detect any individual or small groups of infiltrative cells, and especially any lymphovascular invasion that would exclude Pattern Classification-A.

The differential diagnosis of a Pattern Classification-A tumor includes adenocarcinoma in situ. The distinction between adenocarcinoma in situ and endocervical adenocarcinoma is difficult and can only be made in $80 \%$ of cases. ${ }^{23,24}$ Standard criteria to distinguish invasive endocervical adenocarcinoma from adenocarcinoma in situ include fragmented glands with finger-like projections into the stroma, stromal response, ${ }^{25}$ lymphovascular invasion, ${ }^{9}$ extension of the neoplastic glands beyond the deepest normal endocervical crypt, glands adjacent to thickwalled vessels, ${ }^{26}$ and significant complexity of glands or papillae..$^{8,24,27}$ It should be noted that all of the cases in the current study were originally diagnosed as invasive according to these standard criteria and treated as such. We acknowledge that use of those criteria that do not involve destructive stromal invasive patterns may result in some in situ lesions to be called invasive. We would contend that one of the strengths of the Pattern Classification system is that it obviates these difficulties, as such tumors would be termed either in situ or Pattern Classification-A, and could be treated similarly.

Most prior studies on conservative therapy focused on very small endocervical adenocarcinoma, termed early invasive ${ }^{28}$ or microinvasive. Some use the term microinvasive as equivalent to stage IA1, others for stages IA1 and IA2 regardless of lymphovascular invasion, ${ }^{29}$ and both Society of Gynecologic Oncology ${ }^{30}$ and National Comprehensive Cancer Network as stage IA1 lacking lymphovascular invasion. The rate of nodal disease in microinvasive disease is low, with rates of $<1 \%{ }^{6}$ to $1.5 \% .{ }^{31} \mathrm{~A}$ review of SEER data including 560 cases found no significant differences between stages IA1 and IA2 
endocervical adenocarcinoma in the frequency of positive lymph nodes, recurrence, or death. ${ }^{32}$ One contributor to the similar outcome of stages IA1 and IA2 disease may be the problems associated with the pathological measurement of depth of invasion. This is more problematic for endocervical adenocarcinoma than cervical squamous cell carcinoma as the measured tumor thickness may be used as a substitute for depth of invasion, as previously discussed; this approach is purported to be safer, but actually results in overstaging tumors with an extensive adenocarcinoma in situ component and only a small invasive component. It may be that measuring the 'Depth of Destructive Invasion' (DODI) and carefully noting the presence or absence of lymphovascular invasion would allow translation of the pattern classification used in the current study into clinical practice. Pattern Classification-A endocervical adenocarcinomas have a DODI of 0 , Pattern Classification-B a DODI $<3 \mathrm{~mm}$ and a horizontal extent of DODI $<7 \mathrm{~mm}$, and most but not all Pattern Classification-C show more extensive DODI. Although no patient with a Pattern Classification-A endocervical adenocarcinoma reported to date has been found to have nodal metastases, some tumors that would qualify as Pattern Classification-A (including some initially interpreted as adenocarcinoma in situ) have had ovarian metastases. ${ }^{33,34}$ It may be that Pattern Classification-A tumors should be treated similarly to adenocarcinoma in situ. ${ }^{35}$ In addition, criteria for adjuvant therapy following surgery often include a size $>2 \mathrm{~cm},{ }^{36}$ yet some Pattern Classification-A tumors are $>2 \mathrm{~cm}$ in size; we suggest that pattern classification could also guide the need for adjuvant therapy.

The pattern of invasion affects prognosis in other organs and other histologic subtypes of carcinoma. Intestinal-type mucinous carcinomas of the ovary with expansile or confluent glandular invasion have a significantly better prognosis compared with those with destructive or infiltrative stromal invasion. ${ }^{37,38}$ We had originally discussed whether a two-tiered system of expansile vs destructive stromal invasion could be applied to endocervical adenocarcinoma, as the prognostic implication may be similar. The term expansile, however, did not seem to be an adequate descriptor for Pattern Classification-A. A destructive stromal invasive pattern also is associated with a worsened prognosis for squamous cell carcinoma of the vulva and cervix. ${ }^{39,40}$ Although our studies have focused only on endocervical adenocarcinoma, it may be that similar tumor mechanisms operate for multiple tumor types. Additionally, our findings confirm many other studies indicating that lymphovascular invasion is an indicator of poor prognosis for endocervical adenocarcinoma. ${ }^{20,41}$

The current reproducibility study found an overall concordance of $74 \%$, and substantial agreement with the reference diagnosis was achieved by $86 \%$ of the reviewers. Two studies, including the current, have now shown moderate to substantial reproducibility; ${ }^{4}$ this level of concordance and reproducibility is in the same range as other pathology studies for wellestablished diagnostic categories. ${ }^{11,42,43}$ The study by Paquette et al. ${ }^{4}$ suggested that reproducibility is improved by grouping Pattern Classification-B and $-\mathrm{C}$, and the authors suggested that a two-tier system may be preferable. In the current study, however, the reproducibility of Pattern Classification-A vs Pattern Classification-B+C only achieved the same kappa statistic as that for Pattern Classification-A alone, thus we did not find improved reproducibility with a two-tier system. Additionally we believe the middle category of Pattern Classification-B improves the clinical utility, as Pattern Classification-B tumors without lymphovascular invasion do very well and even Pattern Classification-B tumors with lymphovascular invasion do not behave as aggressively as Pattern Classification-C tumors. Thus, the threetiered pattern classification is recommended.

In summary, the current reproducibility study found overall substantial agreement. Close attention to the presence or absence of lymphovascular invasion, and examination of the entire tumor when initial sections show Pattern Classification-A or Pattern Classification-B, is essential to proper pattern classification. Tumors with overall features of Pattern Classification-A but with small foci of desmoplasia, stromal loosening, or inflammation should be examined carefully at high power, and perhaps with multiple levels to rule out individual or small groups of tumor cells or lymphovascular invasion that would indicate a Pattern Classification-B. The reproducibility of Pattern Classification-C is the highest of the three patterns as diffuse destructive invasion can usually be easily recognized, although further studies on confluence, size criteria, solid growth patterns, and cases that are less than classic may be necessary. We are considering development of a web page with multiple drawing and illustrations of the three patterns. Additional studies on use of DODI, application to endocervical adenocarcinoma variants, and clinical aspects should allow use of this powerful classification system to delineate which endocervical adenocarcinoma can be safely treated conservatively.

\section{Disclosure/conflict of interest}

The authors declare no conflict of interest.

\section{References}

1 Ward KK, Shah NR, Saenz CC et al. Changing demographics of cervical cancer in the United States (1973-2008). Gynecol Oncol 2012;126:330-333.

2 Diaz De Vivar A, Roma AA, Park KJ et al. Invasive endocervical adenocarcinoma: proposal for a new pattern-based classification system with significant clinical implications: a multi-institutional study. Int J Gynecol Pathol 2013;32:592-601. 
3 Roma AA, Diaz De Vivar A, Park KJ et al. Invasive endocervical adenocarcinoma: a new pattern-based classification system with important clinical significance. Am J Surg Pathol 2015;39:667-672.

4 Paquette C, Jeffus SK, Quick CM et al. Interobserver variability in the application of a proposed histologic subclassification of endocervical adenocarcinoma. Am J Surg Pathol 2015;39:93-100.

5 Sedlis A, Bundy BN, Rotman MZ et al. A randomized trial of pelvic radiation therapy versus no further therapy in selected patients with stage IB carcinoma of the cervix after radical hysterectomy and pelvic lymphadenectomy: A Gynecologic Oncology Group Study. Gyn Oncol 1999;73:177-183.

6 http://www.nccn.org/professionals/physician_gls/pdf/ cervical.pdf (accessed on 13 October 2015).

7 American Joint Committee on Cancer. In: Edge SB, Byrd DR, Compton CC et al. (eds). AJCC Cancer Staging Manual, 7th edn. Springer: Chicago, IL, 2010.

8 McCluggage WG. New developments in endocervical glandular lesions. Histopathology 2013;62:138-160.

9 Loureiro J, Oliva E. The spectrum of cervical glandular neoplasia and issues in differential diagnosis. Arch Pathol Lab Med 2014;138:453-483.

10 Landis JR, Koch GG. The measurement of observer agreement for categorical data. Biometrics 1977;33:159-174.

11 Elmore JG, Longton GM, Carney PA et al. Diagnostic concordance among pathologists interpreting breast biopsy specimens. JAMA 2015;313:1122-1132.

12 Galic V, Herzog TJ, Lewin SN et al. Prognostic significance of adenocarcinoma histology in women with cervical cancer. Gynecol Oncol 2012;125:287-291.

13 Suprasert P, Srisomboon J, Charoenkwan K et al. Twelve years experience with radical hysterectomy and pelvic lymphadenectomy in early stage cervical cancer. J Obstet Gynaecol 2010;30:294-298.

14 Kenny SL, McBride HA, Jamison J et al. Mesonephric adenocarcinomas of the uterine cervix and corpus: HPV-negative neoplasms that are commonly PAX8, CA125, and HMGA2 positive and that may be immunoreactive with TTF1 and hepatocyte nuclear factor 1beta. The Am J Surg Pathol 2012;36:799-807.

15 Ito M, Minamiguchi S, Mikami Y et al. Peutz-Jeghers syndrome-associated atypical mucinous proliferation of the uterine cervix: a case of minimal deviation adenocarcinoma ('adenoma malignum') in situ. Pathol Res Pract 2012;208:623-627.

16 Kocken M, Baalbergen A, Snijders PJ et al. High-risk human papillomavirus seems not involved in DESrelated and of limited importance in nonDES related clear-cell carcinoma of the cervix. Gynecol Oncol 2011;122:297-302.

17 Karamurzin YS, Kiyokawa T, Parkash $\mathrm{V}$ et al. Gastric-type endocervical adenocarcinoma: an aggressive tumor with unusual metastatic patterns and poor prognosis. Am J Surg Pathol 2015;39:1449-1457.

18 Kojima A, Mikami Y, Sudo T et al. Gastric morphology and immunophenotype predict poor outcome in mucinous adenocarcinoma of the uterine cervix. Am J Surg Pathol 2007;31:664-672.

19 Winer I, Alvarado-Cabrero I, Hassan $\mathrm{O}$ et al. The prognostic significance of histologic type in early stage cervical cancer-a multi-institutional study. Gynecol Oncol 2015;137:474-478.

20 Baalbergen A, Smedts F, Helmerhorst TJ. Conservative therapy in microinvasive adenocarcinoma of the uterine cervix is justified: an analysis of 59 cases and a review of the literature. Int J Gynecol Pathol 2011;21: 1640-1645.

21 McHale MT, Le TD, Burger RA et al. Fertility sparing treatment for in situ and early invasive adenocarcinoma of the cervix. Obstet Gynecol 2001;98(Pt 1): $726-731$.

22 Ceballos KM, Shaw D, Daya D. Microinvasive cervical adenocarcinoma (FIGO stage $1 \mathrm{~A}$ tumors): results of surgical staging and outcome analysis. Am J Surg Pathol 2006;30:370-374.

23 Jordan SM, Chase DM, Watanabe $\mathrm{T}$ et al. High pathologic misdiagnosis of cervical adenocarcinoma in situ. Eur J Gynaecol Oncol 2013;34:446-449.

24 Zaino RJ. Symposium part I: adenocarcinoma in situ, glandular dysplasia, and early invasive adenocarcinoma of the uterine cervix. Int J Gynecol Pathol 2002;21:314-326.

25 Jordan SM, Watanabe T, Osann K et al. Desmoplastic stromal response as defined by positive $\alpha$-smooth muscle actin staining is predictive of invasion in adenocarcinoma of the uterine cervix. Int J Gynecol Pathol 2012;31:369-376.

26 Wheeler DT, Kurman RJ. The relationship of glands to thick-wall blood vessels as a marker of invasion in endocervical adenocarcinoma. Int J Gynecol Pathol 2005;24:125-130.

27 Zaino RJ. Glandular lesions of the uterine cervix. Mod Pathol 2000;13:261-274.

28 Lee KR, Flynn CE. Early invasive adenocarcinoma of the cervix. Cancer 2000;89:1048-1055.

29 Reynolds EA, Tierney K, Keeney GL et al. Analysis of outcomes of microinvasive adenocarcinoma of the uterine cervix by treatment type. Obstet Gynecol 2010;116:1150-1157.

30 Sevin BU, Nadji M, Averette HE et al. Microinvasive carcinoma of the cervix. Cancer 1992;70:2121-2128.

31 Bisseling KC, Bekkers RL, Rome RM et al. Treatment of microinvasive adenocarcinoma of the uterine cervix: a retrospective study and review of the literature. Gynecol Oncol 2007;107:424-430.

32 Smith HO, Qualls CR, Romero AA et al. Is there a difference in survival for IA1 and IA2 adenocarcinoma of the uterine cervix? Gynecol Oncol 2002;85:229-241.

33 Elishaev E, Gilks CB, Miller D et al. Synchronous and metachronous endocervical and ovarian neoplasms: evidence supporting interpretation of the ovarian neoplasms as metastatic endocervical adenocarcinomas simulating primary ovarian surface epithelial neoplasms. Am J Surg Pathol 2005;29:281-294.

34 Ronnett BM, Yemelyanova AV, Vang R et al. Endocervical adenocarcinomas with ovarian metastases: analysis of 29 cases with emphasis on minimally invasive cervical tumors and the ability of the metastases to simulate primary ovarian neoplasms. Am J Surg Pathol 2008;32:1835-1853.

35 Bull-Phelps SL, Garner EIO, Walsh CS et al. Fertilitysparing surgery in 101 women with adenocarcinoma in situ of the cervix. Gynecol Oncol 2007;107:316-319.

36 Al-Kalbani M, McVeigh G, Nagar H et al. Do FIGO stage IA and small ( $</=2 \mathrm{~cm}$ ) IB1 cervical adenocarcinomas have a good prognosis and warrant less radical surgery? Int J Gynecol Pathol 2012;22:291-295.

37 Lee KR, Scully RE. Mucinous tumors of the ovary: a clinicopathologic study of 196 borderline tumors (of intestinal type) and carcinomas, including an evaluation of 11 cases with 'pseudomyxoma peritonei'. Am J Surg Pathol 2000;24:1447-1464. 
38 Riopel MA, Ronnett BM, Kurman RJ. Evaluation of diagnostic criteria and behavior of ovarian intestinal-type mucinous tumors: atypical proliferative (borderline) tumors and intraepithelial, microinvasive, invasive, and metastatic carcinomas. Am J Surg Pathol 1999;23:617-635.

39 Khunamornpong S, Lekawanvijit S, Settakorn J et al. Prognostic model in patients with early-stage squamous cell carcinoma of the uterine cervix: a combination of invasive margin pathological characteristics and lymphovascular space invasion. Asian Pac J Cancer Prev 2013;14:6935-6940.

40 Jeffus SK, Gehlot A, Holthoff E et al. A fibromyxoid stromal response is associated with an infiltrative tumor morphology, perineural invasion, and lymph node metastasis in squamous cell carcinoma of the vulva. Am J Surg Pathol 2015;39:1226-1233.

41 Poynor EA, Marshall D, Sonoda Y et al. Clinicopathologic features of early adenocarcinoma of the cervix initially managed with cervical conization. Gynecol Oncol 2006;103:960-965.

42 Stoler MH, Schiffman M. Interobserver reproducibility of cervical cytologic and histologic interpretations: realistic estimates from the ASCUS-LSIL Triage Study. JAMA 2001;285:1500-1505.

43 Kendall BS, Ronnett BM, Isacson C et al. Reproducibility of the diagnosis of endometrial hyperplasia, atypical hyperplasia, and well-differentiated carcinoma. Am J Surg Pathol 1998;22:1012-1019. 